\title{
A Patient with a Type I Split Cord Malformation and an Open Myelomeningocele without Advanced Lower Limb Paresis: A Case Report and a Review of the Literature
}

\author{
Miiko ITO ${ }^{1}$, Shinya SATO ${ }^{1}$, and Yukihiko SONODA ${ }^{1}$ \\ ${ }^{1}$ Department of Neurosurgery, Yamagata University, Faculty of Medicine, \\ Yamagata, Yamagata, Japan
}

\begin{abstract}
We report a rare case of a split cord malformation (SCM) combined with an open myelomeningocele (MMC) on the right hemicord. The patient was a male neonate, who exhibited an MMC in the lumbosacral region at birth. Both of his lower limbs moved with slight spasticity, but no atrophic changes or clubfoot deformities were seen. Three-dimensional computed tomography (CT) demonstrated a bony septum, and the patient was diagnosed with a type I SCM. Magnetic resonance imaging (MRI) showed an MMC on the right hemicord (a hemimyelomeningocele). The repair of the open MMC and the removal of the septum were performed immediately to prevent infection and neurological deterioration. Intraoperatively, the right hemicord was thinner than the left hemicord. No additional neurological deficits or complications appeared during treatment. Our findings suggest that when a minor hemicord is affected by both an SCM and an open MMC, good functional outcomes of the lower limbs can be achieved.
\end{abstract}

Keywords: split cord malformation, hemicord, myelomeningocele

\section{Introduction}

A split cord malformation (SCM) is a rare congenital anomaly of the spinal cord. Pang et al. defined two types of SCM: type I SCM involves two hemicords, each contained within its own dural tube, which are separated by a dura-sheathed rigid osseocartilaginous median septum, and type II SCM involves two hemicords housed in a single dural tube, which are separated by a non-rigid, fibrous median septum. ${ }^{1,2)}$ Open myelomeningocele (MMC) is the most common open neural tube defect (ONTD), and it is sometimes associated with SCM. Conversely, approximately $25 \%-35 \%$ of SCM are associated with ONTD. ${ }^{3)}$ It is generally accepted that patients with MMC usually exhibit paraplegia of the lower limbs unless it was not distal end of spinal cord, and bladder and rectal dysfunction. On the other hand, since patients with a combination of a type

Received February 5, 2020; Accepted May 21, 2020

Copyright $\subseteq 2021$ by The Japan Neurosurgical Society This work is licensed under a Creative Commons AttributionNonCommercial-NoDerivatives International License.
I SCM and an open MMC have one impaired hemicord and one functional hemicord, their motor function prognosis is usually better than that of patients with conventional MMC. ${ }^{4-8}$ Here, we report a rare case, in which a myeloschisis was seen on the right hemicord in a patient with type I SCM, who did not exhibit paresis of the lower limbs.

\section{Case Report}

A male infant was diagnosed with hydrocephalus at 26 weeks of gestation based on an intrauterine ultrasound examination. Magnetic resonance imaging (MRI) conducted at 30 weeks of gestation revealed a lumbosacral lesion. The infant was delivered by cesarean section at 38 weeks of gestation. We observed a bulging sac and skin defect in the center of the lumbar region (Fig. 1), but no atrophic changes or clubfoot deformities were seen. A neurological examination revealed slight spasticity of the legs without any apparent paralysis. Three-dimensional computed tomography (3D-CT) revealed spina bifida of the lumbosacral region and bony septum formation just posterior to the L3 vertebral body (Fig. 2A). MRI showed a split 


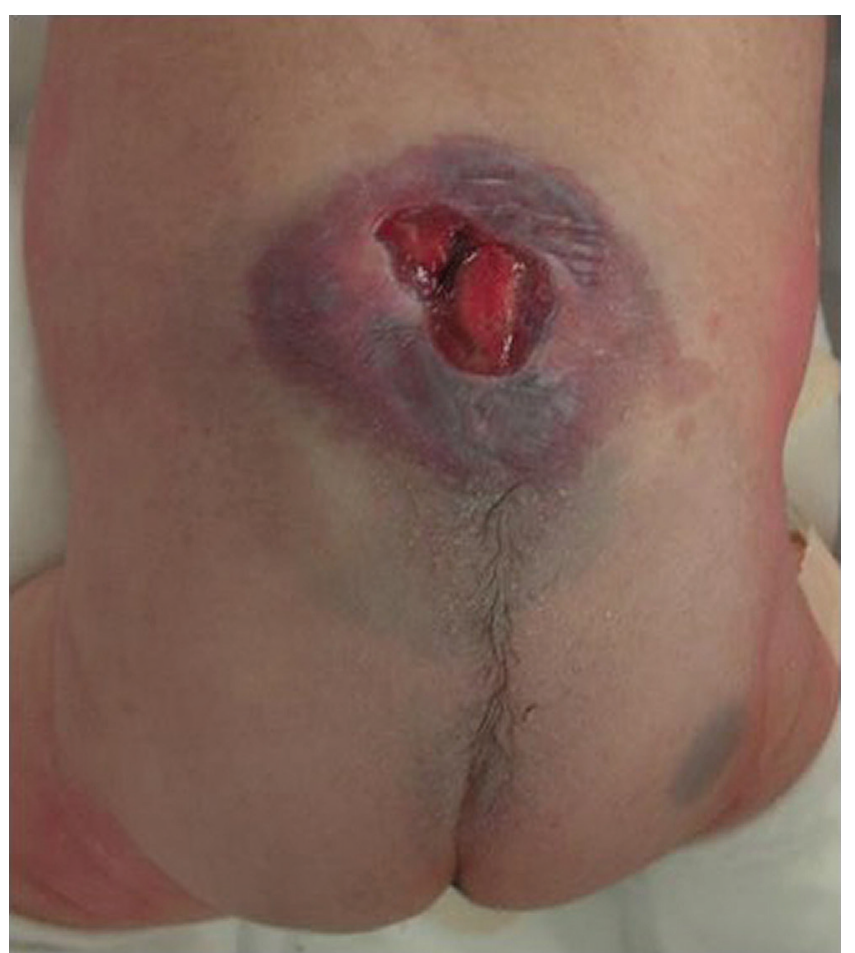

Fig. 1 Photograph showing a MMC in the lumbosacral region. MMC: myelomeningocele.

cord on T2-weighted axial images (Fig. 2B). The preoperative diagnosis was an open MMC associated with a type I SCM.

The patient underwent repair of the open MMC and untethering immediately after birth. An intraoperative examination showed that the spinal cord had been divided rostrally at the L3 level by the bony septum and had fused in the caudal region (Fig. 3A). Myeloschisis was only observed in the right hemicord. The placode was closed, and then the bony septum was removed along with dural sleeve (Figs. 3B and 3C). Generally said, dural closure is unnecessary. But we closed dural defect to prevent the tethering after surgery because the septum was so thick that fatty and bloody fluid exuded from the bone marrow of bony septum. In contrast to the thick and well developed left hemicord and the both side of nerve roots (Figs. 3D and $3 \mathrm{E}$ ), the right hemicord and the right nerve roots were thin and poorly developed (Fig. 3F). During this surgery, electrophysiological monitoring was not performed. The placode, which gradually tapered and combined to the left hemicord on the caudal side (Fig. 3G), and then five-layer closure was performed. He underwent a ventriculoperitoneal shunt for progressive hydrocephalus at 9 days of age.
The patient's postoperative course was uneventful; i.e., no complications or neurological deterioration occurred. Urodynamic study at the age of 1 year and 7 months demonstrated no evidence of bladder dysfunction such as vesicoureteral reflux or poor compliance of bladder. However, neurological examination by pediatric neurologist has revealed that the deep tendon reflex slightly weak and slight flaccid paralysis, both of lower limbs are active in automatic movement in dairy life.

\section{Discussion}

Open MMC are most commonly located caudal to SCM because the open non-neurulated neural placode is usually located at the terminus of the spine. ${ }^{3)}$ Open MMC located at the same level as $\mathrm{SCM}^{4-8,9,10)}$ or rostral to $\mathrm{SCM}^{7,11)}$ are very rare, and, as far as we know, only 10 cases have been reported in the literature (Table 1). In some studies about occult SCM, asymmetric (major and minor) hemicords at the level of SCM were associated with functional discrepancies between the lower limbs. ${ }^{2,12}$ The patients with open MMC complicated SCM had hemiparalysis. ${ }^{4-8)}$ However, the prognosis of the lower limbs in patients with open MMC on the minor hemicord might be better than that of open MMC patients with symmetrical hemicords. It was speculated that if the significant difference in the development between the hemicords, the function would be distributed unevenly. Then the patients complicated open MMC with major hemicord could have paraplesia or nearly paraplesia, on the contrary, with minor hemicord could have no paresis or almost the same. It was unlikely that there was a complete uneven distribution of functions, if hemicords were present on both sides. It was speculated that the prognosis of lower limbs with open MMC complicated SCM depended on the hemicords varying degree of development and the side of MMC. In this case as well, there was a slight difference in the function of the lower limbs, and the nerve roots were also confirmed in the minor hemicord with open MMC, suggesting that the functions of lower limbs were not completely localized.

\section{Conclusion}

We reported a rare case, in which an open MMC occurred at the same level as an SCM. Since the open MMC was associated with the minor hemicord, the prognosis of the lower limbs was better. 

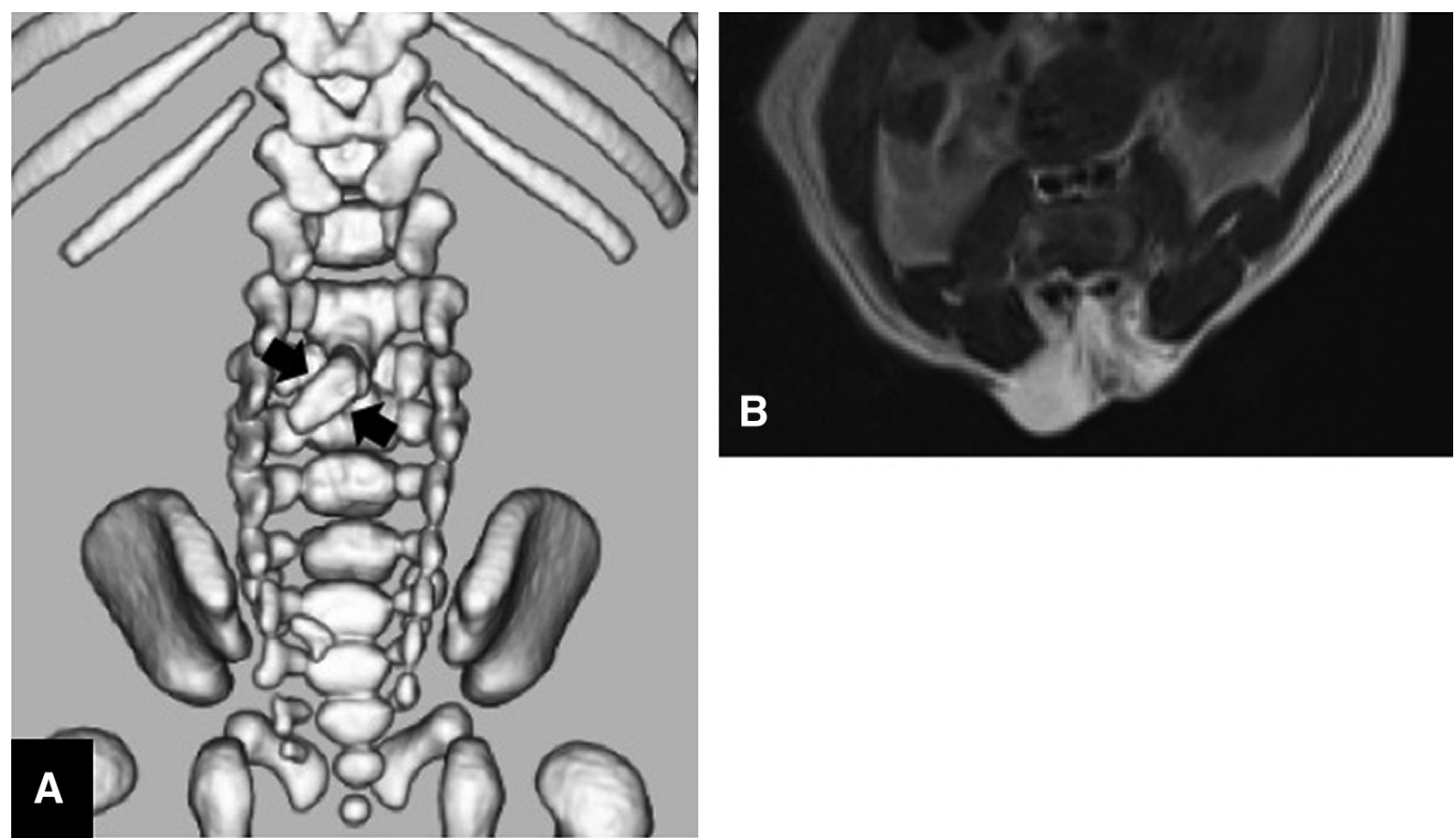

Fig. 2 CT and MRI findings. (A) Three-dimensional CT showed a bony septum (arrow) and spina bifida. (B) Axial MRI showed that the spinal cord had been divided. CT: computed tomography, MRI: magnetic resonance imaging.
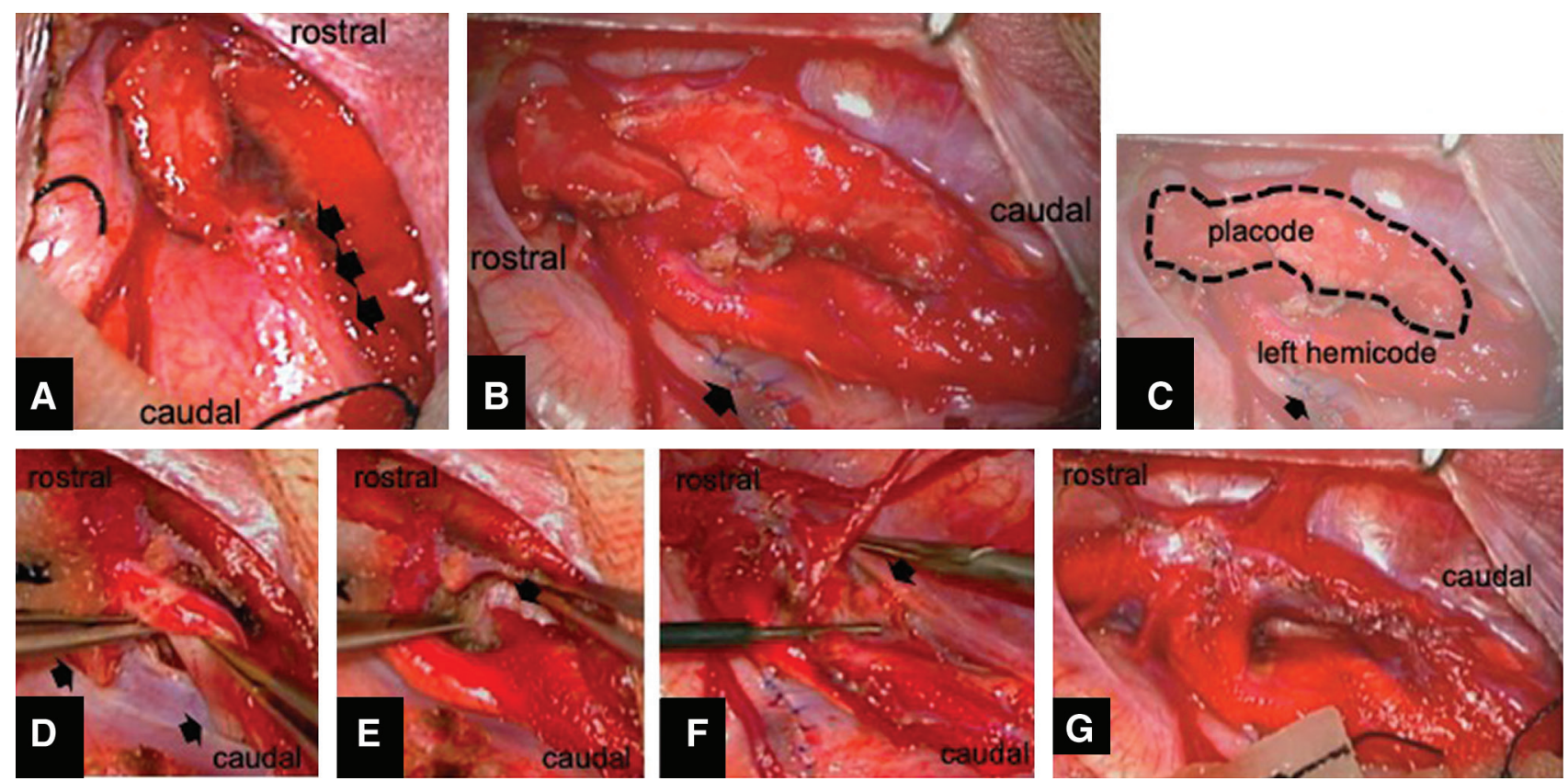

Fig. 3 (A) Intraoperative photographs showing the spinal cord divided by a bone spur (arrows) and myeloschisis on the right hemicord. (B, C) The left hemicord was revealed after the removal of the bony spur along with dural sleeve, and the duraplasty (arrow). (D) The left side of thick nerve roots (arrow) of the left hemicord. (E) The right side of nerve roots (arrow) of the left hemicord. (F) The right side of nerve roots (arrows) of the right hemicord. (G) The right hemicord after repair of placode gradually tapered and combined to the left hemicord on the caudal side. 
Table 1 Summary of reports about MMC located at the same level as or rostral to SCM

\begin{tabular}{rlcclll}
\hline & Author (year) & $\begin{array}{c}\text { Age/ } \\
\text { gender }\end{array}$ & $\begin{array}{c}\text { Type of } \\
\text { SCM }\end{array}$ & $\begin{array}{l}\text { Location } \\
\text { of MMC }\end{array}$ & Hemicord involvement & Lower limb symptoms \\
\hline 1 & Akiyama (2007) & F & 2 & Same level & Both hemicords & Paraplegia \\
2 & Sato (2002) & F & 1 & Same level & One hemicord (symmetrical) & Hemiparesis \\
3 & Yamanaka (2001) & F & 1 & Same level & One hemicord (symmetrical) & Hemiparesis \\
4 & Connolly (2002) & F & 1 & Same level & One hemicord (symmetrical) & Hemiparesis \\
5 & Rowly (2009) & ND & 1 & Same level & One hemicord (symmetrical) & Hemiparesis \\
6 & Higashida (2010) & F & 1 & Same level & One hemicord (symmetrical) & Hemiparesis \\
7 & Jans (2008) & ND & 1 & Same level & One minor hemicord (asymmetrical) & No paresis \\
8 & Higashida (2010) & F & 1 & Same level & One minor hemicord (asymmetrical) & No advanced paresis \\
9 & Higashida (2010) & F & 2 & Same level & N/A & Paraplegia \\
10 & Addas (2014) & 49 & 1 & Rostral & N/A & Paraplegia \\
11 & present case & $\mathrm{M}$ & 1 & Same level & One minor hemicord (asymmetrical) & No advanced paresis \\
\hline
\end{tabular}

F: female, MMC: myelomeningocele, NA: not applicable, ND: not described, SCM: split cord malformation.

\section{Conflicts of Interest Disclosure}

The authors declare that they have no conflicts of interest associated with this manuscript.

\section{References}

1) Pang D, Dias MS, Ahab-Barmada M: Split cord malformation: Part I: A unified theory of embryogenesis for double spinal cord malformations. Neurosurgery 31: 451-480, 1992

2) Pang D: Split cord malformation: Part II: clinical syndrome. Neurosurgery 31: 481-500, 1992

3) Pang D: 34 split cord malformation: from gastrulation to operation, in Cohen AR: Pediatric Neurosurgery: Tricks of the Trade, New York, Thieme, 2016, pp. 293-305

4) Rowley VB, Johnson AJ: Lumbar split cord malformation with lateral hemimyelomeningocele and associated Chiari II malformation and other visceral and osseous anomalies: a case report. J Comput Assist Tomogr 33: 923-926, 2009

5) Yamanaka T, Hashimoto N, Sasajima H, Mineura K: A case of diastematomyelia associated with myeloschisis in a hemicord. Pediatr Neurosurg 35: 253-256, 2001

6) Connolly BH, Kasser RJ: Rehabilitation of a child with a split cord malformation and hemimeningomyelocele. Pediatr Phys Ther 14: 208-213, 2002
7) Higashida T, Sasano M, Sato H, Sekido K, Ito S: Myelomeningocele associated with split cord malformation type I-three case reports. Neurol Med Chir (Tokyo) 50: 426-430, 2010

8) Sato K, Yoshida Y, Shirane R, Yoshimoto T: A split cord malformation with paresis of the unilateral lower limb: case report. Surg Neurol 58: 406-409; discussion 409, 2002

9) Jans L, Vlummens P, Van Damme S, Verstraete K, Abernethy L: Hemimyelomeningocele: a rare and complex spinal dysraphism. JBR-BTR 91: 198-199, 2008

10) Akiyama K, Nishiyama K, Yoshimura J, Mori H, Fujii Y: A case of split cord malformation associated with myeloschisis. Childs Nerv Syst 23: 577-580, 2007

11) Addas BM: Split cord malformation type I distal to segmental myelomeningocele. Saudi Med J 35(Suppl 1): S72-S74, 2014

12) Shimokawa S, Hayashi T, Nomura S, Sakamoto K: Split cord malformation: a case report. Nervous System in Children 28: 355-358, 2003 (Japanese)

Corresponding author: Yukihiko Sonoda, MD, PhD Department of Neurosurgery, Yamagata University, Faculty of Medicine, 2-2 Iidanishi, Yamagata, Yamagata, 9909585, Japan.

e-mail: ysonoda@med.id.yamagata-u.ac.jp 